\title{
Time-Resolved Solution Scattering for Structural Biology Research at SSRL BL4-2
}

\author{
Thomas M Weiss, Tsutomu Matsui, Ivan Rajkovic, Ping Liu
}

Stanford Synchrotron Radiation Light Source, Stanford Linear Accelerator Center National Laboratory, Menlo Park, California

Email Contact: weiss@slac.stanford.edu

With time-resolved small angle X-ray scattering (TR-SAXS) in solution we can measure and follow in-situ the conformational changes in macromolecular systems initiated by environmental changes closely mimicking those happening during their physiological lifecycle. The time regime for such changes is generally dependent on the scale of the event under investigation. Fast mixing of two reagent solutions, e.g. in a stopped-flow mixer, is an extremely versatile and ideally suited method to investigate large scale transitions like tertiary or quaternary structural changes of macromolecules typically occurring in the millisecond time scale and above. The use of TR-SAXS experiments for biological samples is however often difficult due to the large amount of material needed for the experiment. Here we report on the latest status of our fast TR-SAXS setup using a customized stopped-flow apparatus optimized for low sample consumption. This new setup allows us to obtain a TR-SAXS data set from as little as 30ul of sample volume. It eliminates the sample consuming priming of internal flow lines and thus substantially reduces the sample amount required for such experiments. Furthermore, the sample cell can be thoroughly cleaned in between shots to ensure no residual sample remains inside from the previous measurement. Recent developments and scientific results using this setup will be presented. 